\title{
Computation of Noise from Homogeneous Turbulence and from a Free Jet
}

\author{
Seungbae Lee ${ }^{(1)}$, William C. Meecham ${ }^{(2)}$ \\ ${ }^{(1)}$ Department of Mechanical Engineering, Inha University, \#253 Yonghyun-Dong Nam-Ku Inchon, \\ KOREA ${ }^{(2)}$ Department of Mechanical and Aerospace Engineering, School of Engineering and Applied \\ Science, University of California, Los Angeles, California 90024, USA
}

\begin{abstract}
Acoustic radiation from turbulent fluids (aeroacoustics) has been a major research area since the pioneering work of Lighthill. We present here a new application of Large-Eddy Simulation (LES) techniques to the calculation of the acoustic far field produced by turbulent fluids. A direct numerical simulation is limited to mean flow Reynolds numbers of about 1500; see the heroic efforts of Mitchell et al. (1995) (clearly useful for many purposes, dealing with a Karman-vortex-street-like structure). This is a limitation set by both computer memory and computation time; there is little realistic prospect of relieving this handicap in the near future. The acoustic radiation problem consists of two steps; first one simulates the turbulent flow field using LES, and in the second step one generates the acoustic far field using Lighthill's procedure (the flow field as source). We first consider statistically homogeneous and isotropic turbulence found using LES employing three subgrid-scale models; the Smagorinsky model, a scale-similarity model, and a deductive model (based on a Taylor series expansion with no adjustable constants), and their combinations. A model which is a combination of the deductive and Smagorinsky model shows the most satisfactory features using as criteria: correlation, decay behavior, and energy spectra. The quadrupole directivity pattern from a few eddies is clearly seen. Of course the use of a time average would remove many of these details; we show first one member of the statistical ensemble. As a second example we treat the turbulent subsonic jet issuing from a square nozzle. A similar calculation for jet noise is performed. The combined subgrid model proposed here avoids a demanding integration process for filtering. This model is used in the simulation of the turbulent jet. In these aerosound calculations of the turbulent jet, the major source region is seen to be the end region of the potential core. The turbulence intensity (as found in experiments) is about $10 \%$, independent of the initiating level. A rapidly changing quadrupole with a peak near 45 degrees from the axis of the jet is obtained. The sound pressures are, within the approximation of order of magnitude estimates, of the size of the Lighthill values, with a multiplicative constant set by the results of many experiments. The jet Reynolds number is 48,000 , fully developed; there is no reason why the procedure cannot be extended well beyond such values. It is noted that the Reynolds number regime is far from that of vortex pairing and the like. Preliminary versions of this work have been presented in several symposia, the first being Lee and Meecham (1992).
\end{abstract}

\section{INTRODUCTION}

Turbulent flows have been the subject of the experimental and theoretical studies since the last century. The variety of turbulent flows is enormous and knowledge of them has many important engineering applications. The instantaneous flow variables satisfy the Navier-Stokes equations.

The strategies for dealing with turbulence are many: turbulence models (educated guesses about the needed Reynolds stresses), statistical theories of turbulence (to gain fundamental understanding), direct numerical simulation of various kinds.

The LES is one of the more promising modes of numerical simulation of turbulence. Most of the developments in the LES approach, except recent ones, have been reviewed by Rogallo and Moin (1984). One of the main differences between the conventional method and the LES technique is the averaging process used in the Navier-Stokes equations. The LES approach does not include the use of ensemble averages as a first step in obtaining equations for mean flows, but uses a space filtering operation applied to the equations of motions, which will be fully discussed in Section 2. One hopes to obtain fluctuations using the LES procedure (which is not the case for turbulence models e.g. $k-\varepsilon$ [see Runchal (1991)]; and of course it is the fluctuations which are responsible for sound production).

We propose here (previously reported, Lee and Meecham (1992)) to use the LES algorithm to calculate aerosound. In large-eddy simulations, one calculates directly the large-scale turbulent motions with a relatively coarse time-dependent, three-dimensional computation using the subgrid-scale (SGS) model for the effects of the small scale motions upon the large scale ones. The basic motivation for this approach is that experimental observations of turbulent flow show that the large turbulent structures differ markedly from one flow to another, but the small-scale turbulent structures are statistically quite similar.

Van Atta (1991) shows that spectral isotropy, as scrutinized in detail by using the spectra of scalars and velocity gradients, is indeed a property of the finest scales of turbulence in many fluid flows. So we may hope to use universal parameters to characterize the subgrid-scale effects and such a treatment should improve as the SGS length and time scales 